\title{
Software Defined Networking for Organizations Network Automation
}

\author{
Diaz-Martinez Jorge Luis $^{1 s t}$, De-La-Hoz-Franco Emiro ${ }^{2 n d}$, Johan David Mardini Bovea ${ }^{\text {3rd }}$, \\ Shariq Aziz Butt ${ }^{4 \text { th }}$, Tauseef Jamal ${ }^{\text {th }}$

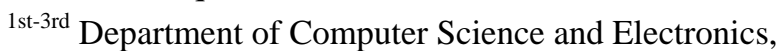 \\ Universidad de la Costa, Barranquilla, Colombia. \\ 4-5th PIEAS University CS Department, Islamabad, Pakistan \\ 1st jdiaz5@cuc.edu.co \\ 2nd edelahoz@cuc.edu.co \\ 3rd johanmardini@mail.uniatlantico.edu.co \\ 4th Shariq2315@gmail.com \\ 5th tauseef.pieas@gmail.com
}

\begin{abstract}
When new applications are deployed, virtual servers or dynamic applications are moved, new instances are launched where the networks must be able to respond immediately and provide the right kind of connectivity. That's why in recent years, adoption of software-based networks (SDNs) and growing interest in defined data centers (SDDCs) have driven a switch from traditional hardware-based networks to networks Based on the software thus enabling organizations to implement this trend to improve network agility by incorporating network automation into cloud-based technology platforms. While many organizations are still in the process of change, the adoption of software-based networks is already occurring. Increasingly, the software-based model of networks is established as a key resource to achieve the adaptation and simplification of changes in network components. However, in order to achieve this migration, it is necessary for organizations to travel on a path that requires evolution.
\end{abstract}

KEYWORDS: SDN (Software Defined Networking), ONF (Open Networking Foundation), Cloud Computing, OpenFlow Protocol.

\section{Introduction:}

The systems (Internet) has a few changes and advancements to fulfill the developing need for applications and different user's services by increasing services such as social network, Mobile services, virtualization services, Cloud Computing-based systems etc., thus there is a need to restructure traditional architectures of network to meet the crowd traffic of users [1]. Conventional system designs present impediments to these new necessities, for example, constrained capacity to adjust to new innovations, the versatility of systems and the un-proficient utilization of access control strategies regularly make security gaps vulnerable to assaults. This has incited a scan for a few options to replace traditional networks. One of these alternatives, from the Open Networking Foundation (ONF), raises the use of Software Defined Networks (SDN) to meet the current requirements of Internet users [1], [2]. The SDN's according to [1] [2] and [3] constitute a network architecture whose fundamental objective is to un-couple the control plane from the data plane, which facilitates a greater control and level of management over the connectivity equipment, guaranteeing the network administrator a centralized control. The term centralized control of the network has clearly logical connotation and therefore, the administration of the network can be centered on one or more controllers that is a control distributed in a physical manner there may even be backup servers in case of a failure of a certain server As a consequence of a centralized and direct control it is possible to enhance the security strategies of a specific substance or organization respond rapidly to changes in the prerequisites of the system or to an adjustment of new innovations and accomplish exceedingly versatile systems.

\section{Concept:}

The term SDN (Software Define Network or software Define network) according to [1] [4] is Define as the network architecture that allows to separate the control plane from the data plane to achieve more programmable, scalable and automatable network thus allowing accelerate the implementation and distribution of applications, greatly reducing cost through the automation of workflow based on policies. Characterized in the enabling of architectures in the cloud (Cloud Computing) through the distribution and mobility of applications and services in an automated way increasing the benefits of virtualization of data centers, since they increase flexibility the use of resources for reducing expenses for infrastructure. According to [5] software Define networks (SDNs) allows organizations to accelerate applications deployment and deployment by drastically reducing IT costs by automation policy-based workflow. SDN technology enables cloud architectures through applications distribution and mobility in an automated, on-demand, and scale manner. SDNs increase the benefits of data center virtualization, as they increase flexibility and resources utilization and reduce overhead and infrastructure costs. To achieve these business objectives SDNs converge the administration of 
network services and applications into centralized and scalable coordination stages that can robotize the provisioning and setup of the whole framework. Basic concentrated IT strategies bind together dissimilar gatherings and IT work process. The outcome is a cutting edge framework that can conveyed new applications and administrations in minutes, rather than days or weeks as before SDN systems concurring to [6] [7] substitute the control level of the network hardware for a software layer infrastructure based on virtualization techniques thus making the network more programmable Characterized by providing access to hardware based on programming through protocols such as OpenFlow and in the creation of virtual networks above the hardware that direct traffic through physical networks.

\section{Normalization of SDN:}

The organization ONF (Open Network Foundation) [3], which leads the evolution and normalization of critical elements (architecture and protocol) of SDN and other organizations such as (ATIS, Broadband Forum, ETSI, OIF, ITU-T, etc.) especially the IETF, through the group the I2RS group (Interface to the Routing System), are also working on extending their specifications to support SDN principles. In addition to the standardization bodies, there are several open source initiatives, such as Open Stack, which are specifying several SDN tools. Entities such as ETSI [7] and ONF [3,8] have collaborated since the creation of NFC ISG in 2012 developing strong collaborations to promote the development of the NFV specifications, which accelerates the adoption of NFV as SDN for their coordinated development. The aforementioned organizations thus show their commitment to support the requirements of the operators and a complementary approach in the development of standards, methodologies and knowledge sharing. ETSI NFV ISG will promote concept tests that use both NFV and SDN to demonstrate the benefits of using both technologies together. The ONF organization has developed a technical report that clearly shows how IT operators are combining NFV and SDN [8] [9] to achieve common goals of both technologies to improve the agility of the network, in turn explains the challenges that operators have to overcome for the effective implementation of NFV and presents some cases that demonstrate SDN enabled by Open Flow can satisfy the needs of a more programmable and flexible network to support NFV.

\section{SDN ARCHITECTURE:}

The SDN architecture according to [1] is constituted by 3 (three) layers as shown in fig. 1 and is specified as follows:

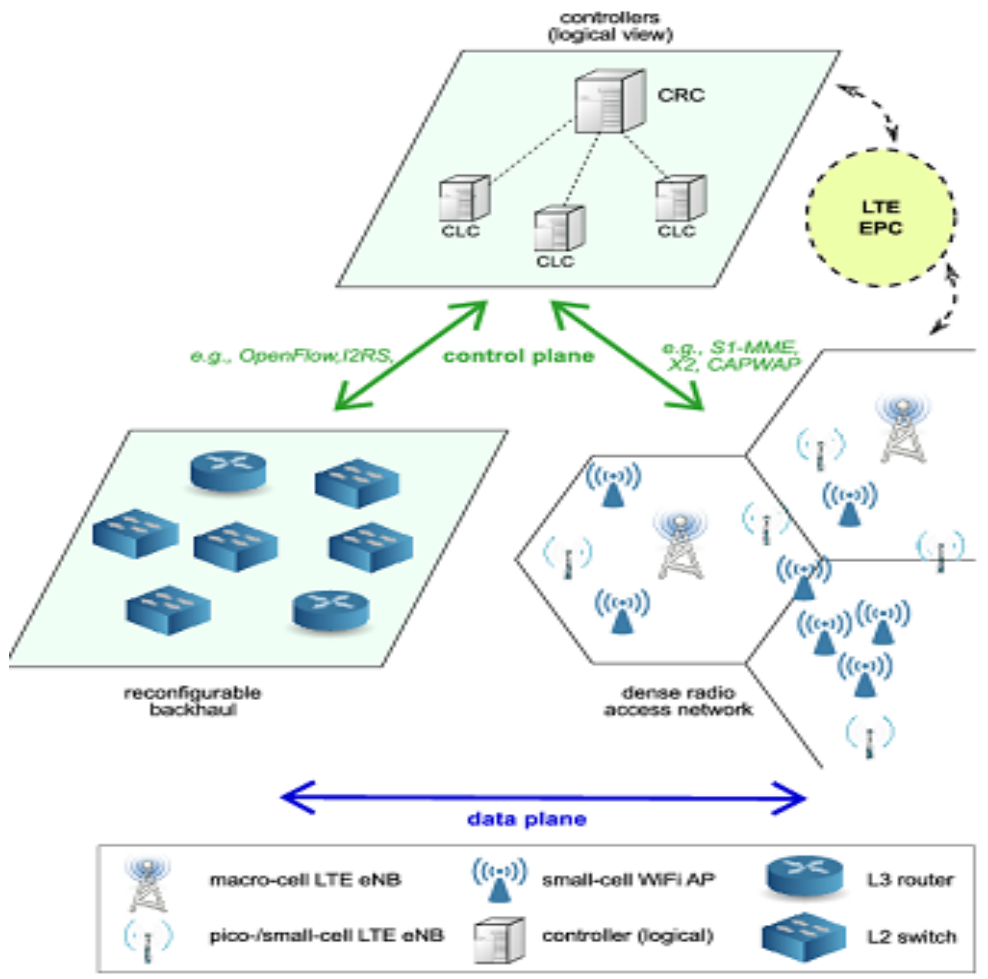

Fig. 1. The architecture of SDN [1]

\subsection{Infrastructure layer:}

Consisting of network nodes that perform packet routing and routing, providing programmable open access under API down (southbound), as in the case of Open Flow. 


\subsection{Control layer:}

The SDN controller is a software entity that has exclusive control over an abstract set of control plane resources, that is the entity that controls and configures the network nodes to correctly direct the traffic flows. The SDN controller eliminates the intelligence of switching and routing of data of the nodes that perform this function, passing to the SDN controller, which makes those decisions and selects the best path for traffic. The architecture describes a series of functions internal to the SDN controller and to the network element, but only the behavior of those aspects that are necessary to ensure interoperability is specified. The architecture is agnostic to the protocols used between interfaces. The architecture allows an SDN controller to manage a wide range of data plane resources, which offers the potential to unify and simplify its configuration.

\subsection{Application layer:}

It is made up of the applications for end users, which use the SDN communication services through the APIs upwards (northbound) of the control layer, such as JSon, REST, among others, which allows the services and applications to simplify and automate the tasks of configuration, provisioning and managing new services in the network, thus generating new routes for revenue and innovation in infrastructure.

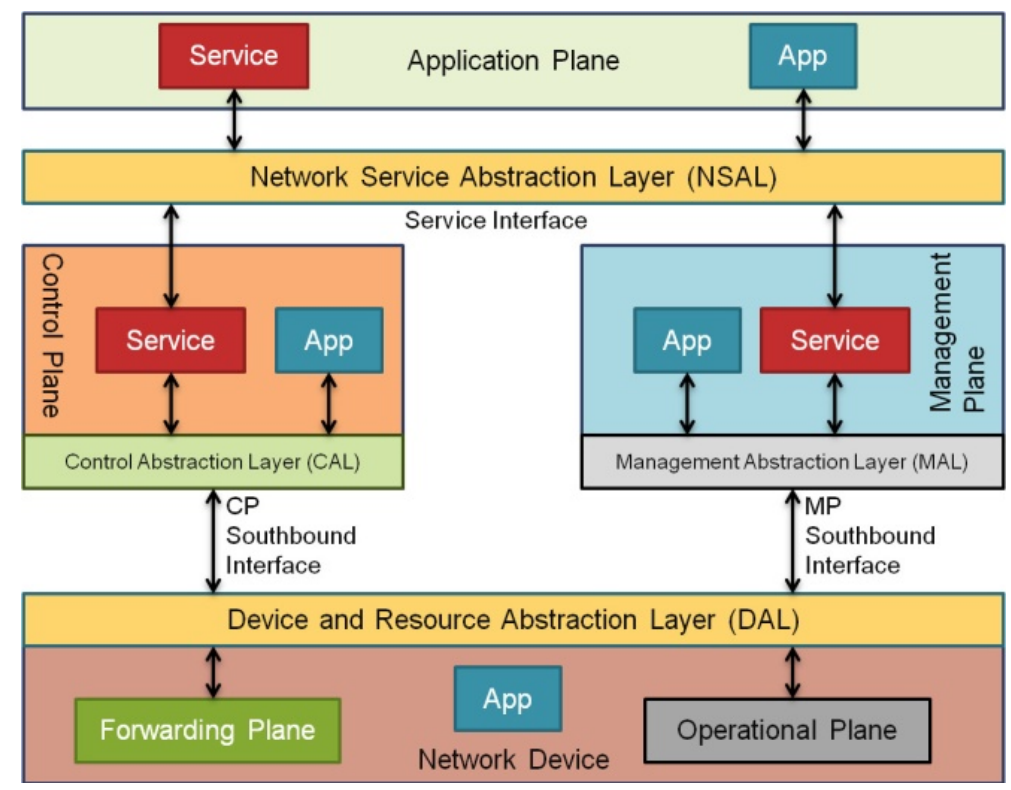

Fig.2 Layers in SDN [10]

\section{STANDARD OpenFlow:}

As per [11], the most famous particular for making a product characterized system is an open standard called OpenFlow, which permits arrange overseers to control directing tables remotely, along these lines getting to be one of the principal correspondence interface measures characterized. between the control and sending layers in an SDN engineering. Also, it permits direct access and control of the sending layer of system gadgets, regardless of whether virtual or physical. This convention is actualized on the two sides of the interface, between the system foundation and the SDN control programming utilizing the stream idea to recognize arrange traffic dependent on the gathering of predefined rules, which can be static or dynamic, programmable through SDN control programming [11, 18, 19]. According to [10, 12, 17], it forms the basis of open networks defined by standards-based software with collaboration from the academic and business sectors, with the Stanford and California universities in Berkeley leading the reins in the first instance. Currently, the Open Networking Foundation (ONF) is responsible for defining the standard.

\section{ADVANTAGES OF SDN IMPLEMENTATION:}

The main objective according to $[13,14]$, the networks defined by software is the reduction of expenses for equipment and network operation, in turn increasing the speed of innovation to boost an open system, accelerating the introduction of new services and above all allowing Agile form scalability creating new business opportunities with their customers by offering them access to information about the state of the network and the management of their traffic flows more efficiently and more intelligently.

To obtain the maximum performance, utilization and simplicity, telecommunications operators, cloud service providers and companies can use SDN technology in the entire network, from the data center to the desktop, separating the hardware infrastructure from the plan of control, and applications this will solve the complexities that exist today and improve the agility of the business. 
To respond to the challenges generated by conventional networks, organizations need to be able to automate the network from end to end and SDN makes it possible to make the control plane of the physical infrastructure independent.

According to $[14,16,19]$ SDN technology is a flexible and scalable architecture deploying in a platform of applications and services able to respond quickly to changes in the needs of customers of a business, market or end user, where applications can demand resources network in real time, as bandwidth and quality of service can be adapted to services applied to the future.

\section{CONCLUSION:}

Companies are faced daily with the challenges of reducing the risks of implementing services, accelerating implementation times, and reducing operating expenses while giving rise to management in a general IT team. Secondly, companies need to incorporate a solution that allows them to support changes in customer services, facilitate network administration and maximize the use of resources. Software-based networks are the answer to address these challenges.

Software-based networks can be programmed directly and allow administrators to adjust the traffic flow of the network to meet changing needs which makes them a spry model. Then again, it is described as a concentrated innovation that can be arranged and improve assets rapidly, through computerized projects that can be composed independent from anyone else, without depending on exclusive programming. These kinds of systems depend on open and unbiased gauges which disentangle organize tasks and plans, on the grounds that the guidelines are given by the system controllers, and not by various conventions and seller explicit gadgets. Taking everything into account, programming based systems are dynamic, sensible, financially savvy and versatile.

\section{References:}

[1] Ali-Ahmad, Hassan, et al. "An SDN-based network architecture for extremely dense wireless networks." Future Networks and Services (SDN4FNS), 2013 IEEE SDN for. IEEE, 2013.

[2] Jamal, Tauseef, Pedro Amaral, and Khurram Abbas. "Flow Table Congestion in Software Defined Networks." ICDS 2018(2018): 57.

[3] Jain, Raj, and Subharthi Paul. "Network virtualization and software-defined networking for cloud computing: a survey." IEEE Communications Magazine 51.11 (2013): 24-31.

[4] Chen, Tao, et al. "Software-defined mobile networks: concept, survey, and research directions." IEEE Communications Magazine 53.11 (2015): 126-133.

[5] Yi, Shane, Cheng Li, and Qun Li. "A survey of fog computing: concepts, applications and issues." Proceedings of the 2015 workshop on mobile big data. ACM, 2015.

[6] Porras, Philip, et al. "A security enforcement kernel for OpenFlow networks." Proceedings of the first workshop on Hot topics in software defined networks. ACM, 2012.

[7] Bakshi, Kapil. "Considerations for software-defined networking (SDN): Approaches and use cases." Aerospace Conference, 2013 IEEE. IEEE, 2013.

[8] Janz, Christopher, et al. "Emerging transport Sdn architecture and use cases." IEEE Communications Magazine 54.10 (2016): 116-121.

[9] Matias, Jon, et al. "Toward an SDN-enabled NFV architecture." IEEE Communications Magazine 53.4 (2015): 187-193.

[10] Haleplidis, Evangelos. "Overview of RFC7426: SDN Layers and Architecture Terminology." IEEE 2017.

[11] Ishimori, Airton, et al. "Control of multiple packet schedulers for improving QoS on OpenFlow/SDN networking." Software Defined Networks (EWSDN), 2013 Second European Workshop on. IEEE, 2013.

[12] Padma, V., and P. Yogesh. "Proactive failure recovery in OpenFlow based software-defined networks." Signal Processing, Communication, and Networking (ICSCN), 2015 3rd International Conference on. IEEE, 2015.

[13] Jarschel, Michael, et al. "Interfaces, attributes, and use cases: A compass for SDN." IEEE Communications Magazine 52.6 (2014): 210-217.

[14] De Oliveira, Rogério Leão Santos, et al. "Using mini net for emulation and prototyping software-defined networks." 2014 IEEE Colombian Conference on Communications and Computing (COLCOM). IEEE, 2014.

[15] Doverspike, Robert, et al. "Using SDN technology to enable cost-effective bandwidth-on-demand for cloud services." Journal of Optical Communications and Networking 7.2 (2015): A326-A334.

[16] Jamal, T., \& Mendes, P. (2010, October). Relay selection approaches for wireless cooperative networks. In 2010 IEEE 6th International Conference on Wireless and Mobile Computing, Networking and Communications (pp. 661-668). IEEE.

[17] Jamal, T., Haider, Z., Butt, S. A., \& Chohan, A. (2018). Denial of Service Attack in Cooperative Networks. arXiv preprint arXiv:1810.11070.

[18] Jamal, T., \& Butt, S. A. (2017). Cooperative Cloudlet for Pervasive Networks. Proc. of Asia Pacific Journal of Multidisciplinary Research, 5(3), 42-26.

[19] Jamal, T., Amaral, P., Khan, A., Zameer, A., Ullah, K., \& Butt, S. A. (2018). Denial of Service Attack in Wireless LAN. ICDS 2018, 51. 